\title{
Incremental diagnostic role of left atrial strain analysis in thrombotic risk assessment of nonvalvular atrial fibrillation patients planned for electrical cardioversion
}

\author{
Andrea Sonaglioni ${ }^{1} \cdot$ Michele Lombardo $^{1} \cdot$ Gian Luigi Nicolosi ${ }^{2} \cdot$ Elisabetta Rigamonti $^{1} \cdot$ Claudio Anzà $^{3}$
}

Received: 25 October 2020 / Accepted: 30 November 2020 / Published online: 3 January 2021

(c) The Author(s), under exclusive licence to Springer Nature B.V. part of Springer Nature 2021

\begin{abstract}
During the COVID-19 pandemic, transesophageal echocardiography (TEE) for left atrial appendage thrombosis (LAAT) detection should be limited to situations of absolute necessity. We sought to identify the main conventional and functional echocardiographic parameters associated with LAAT on TEE in non-valvular atrial fibrillation (NVAF) patients planned for electrical cardioversion (ECV). This retrospective study included 125 consecutive NVAF patients ( $71.5 \pm 7.8 \mathrm{yrs}, 75$ males), who underwent TEE at our Institution between April 2016 and January 2020, to exclude LAAT before scheduled ECV. All patients underwent a transthoracic echocardiography (TTE) implemented with speckle tracking echocardiography (STE) analysis of left atrial (LA) strain and strain rate (SR) parameters. 28\% of patients were diagnosed with LAAT, while $72 \%$ without LAAT. Compared to controls, patients with LAAT had significantly higher $\mathrm{CHA}_{2} \mathrm{DS}_{2}$-Vasc Score and average E/e' ratio, and significantly lower left ventricular ejection fraction (LVEF). Moreover, LA-peak positive global atrial strain $(\mathrm{GSA}+)$ and LA-SR parameters were significantly reduced in patients with LAAT. Multivariate logistic regression revealed that, differently from $\mathrm{CHA}_{2} \mathrm{DS}_{2}$-Vasc Score, LVEF (OR 0.88, 95\%CI 0.81-0.97, $\mathrm{p}=0.01$ ), average E/e' ratio (OR 2.36, 95\% CI 1.41-3.98, $\mathrm{p}=0.001)$, and LA-GSA+ (OR 0.57, 95\% CI 0.36-0-90, $\mathrm{p}=0.01)$ were independently associated with LAAT. LA-GSA+ (optimal cut-off $\leq 9.1 \%$, AUC 0.95) showed the highest diagnostic performance. Finally, a strong linear correlation of LA peak-to-peak SR with both LA appendage filling $(r=0.86)$ and emptying $(r=0.83)$ velocities was demonstrated. TTE implemented with STE analysis of LA mechanics improves thrombotic risk assessment of NVAF patients.
\end{abstract}

Keywords COVID-19 · Non-valvular atrial fibrillation · Electrical cardioversion $\cdot$ Left atrial appendage thrombosis $\cdot$ Left atrial strain

\begin{tabular}{|c|c|c|c|}
\hline \multicolumn{2}{|c|}{ Abbreviations } & CI & Confidence interval \\
\hline $2 \mathrm{D}$ & Two-dimensional & COVID-19 & Coronavirus disease 2019 \\
\hline $\mathrm{AF}$ & Atrial fibrillation & $\mathrm{CT}$ & Computed tomography \\
\hline AUC & Area under the curve & ECG & Electrocardiogram \\
\hline \multirow[t]{2}{*}{ BSA } & Body surface area & $\mathrm{ECV}$ & ELECTRICAL cardioversion \\
\hline & & GSA+ & Peak positive global atrial strain \\
\hline \multirow{3}{*}{\multicolumn{2}{|c|}{$\begin{array}{l}\text { Supplementary Information The online version of this article } \\
\text { (https://doi.org/10.1007/s10554-020-02127-6) contains } \\
\text { supplementary material, which is available to authorized users. }\end{array}$}} & GSR+ & Peak positive global atrial strain rate \\
\hline & & GSR- & Negative global atrial strain rate \\
\hline & & ICC & Intraclass correlation coefficient \\
\hline \multirow{2}{*}{$\triangle \mathrm{A}$} & lioni & LA & Left atrial/left atrium \\
\hline & rea@gmail.com & LAA & Left atrial appendage \\
\hline \multirow{2}{*}{$\begin{array}{l}\mathrm{D} \\
\mathrm{N}\end{array}$} & f Cardiology, Ospedale San Giuseppe & LAA-EV & Left atrial appendage-emptying velocity \\
\hline & IRCCS, Via San Vittore 12, 20123 Milan, Italy & LAA-FV & Left atrial appendage-filling velocity \\
\hline \multirow{2}{*}{$\begin{array}{l}\mathrm{I} \\
\mathrm{F}\end{array}$} & f Cardiology, Policlinico San Giorgio, & LAAT & Left atrial appendage thrombosis \\
\hline & & LAVi & Left atrial volume index \\
\hline \multirow[t]{2}{*}{3} & Ir Department, MultiMedica IRCCS, Via & LV & Left ventricular \\
\hline & 20099 Sesto San Giovanni (MI), Italy & LVEF & Left ventricular ejection fraction. \\
\hline
\end{tabular}




$\begin{array}{ll}\text { LVMi } & \text { Left ventricular mass index } \\ \text { MR } & \text { Mitral regurgitation } \\ \text { MRI } & \text { Magnetic resonance imaging } \\ \text { NVAF } & \text { Non-valvular atrial fibrillation } \\ \text { OR } & \text { Odds ratio } \\ \text { ROC } & \text { Receiver operating characteristics } \\ \text { SEC } & \text { Spontaneous echo contrast } \\ \text { SR } & \text { Strain rate } \\ \text { SARS-CoV-2 } & \text { Severe acute respiratory syndrome coror } \\ & \text { virus 2 } \\ \text { STE } & \text { Speckle tracking echocardiography } \\ \text { TEE } & \text { Transesophageal echocardiography } \\ \text { TGSA } & \text { Total global atrial strain } \\ \text { TTE } & \text { Transthoracic echocardiography } \\ \text { VKAs } & \text { Vitamin K antagonists }\end{array}$

\section{Introduction}

Non-valvular atrial fibrillation (NVAF) is associated with increased risk of stroke or systemic embolism [1] and death [2].

In patients presenting with NVAF, over $90 \%$ of thrombi originate into the left atrial appendage (LAA) [3].

Presently, the gold standard for LAA thrombosis (LAAT) detection is two-dimensional (2D) transesophageal echocardiography (TEE) [4].

However, during the coronavirus disease 2019 (COVID19) pandemic, 2D-TEE should be limited to situations of absolute necessity $[5,6]$.

The potential role of $2 \mathrm{D}$ transthoracic echocardiography (TTE) in the risk stratification of NVAF patients and in the prediction of left atrial (LA) stasis has been overlooked [4].

2D speckle tracking echocardiography (STE) is a relatively new, noninvasive method for the assessment of cardiac function. It provides accurate and angle-independent information also regarding LA myocardial deformation and motion [7]. LA functional assessment by 2D-STE has been employed in different clinical settings [8-10].

Including LA strain analysis to the conventional 2D-TTE evaluation of NVAF patients scheduled for electrical cardioversion (ECV) may provide more complete information on LA mechanics and may improve thromboembolic risk assessment.

To date, there are a limited number of studies that have evaluated LA myocardial strain with 2D-STE analysis in NVAF patients [11-14].

The aim of this study was to identify the main conventional and functional echocardiographic parameters associated with the presence of LAAT on 2D-TEE in NVAF patients planned for ECV.

\section{Methods}

\section{Patient selection and clinical measures}

This retrospective observational monocentric study included all patients with persistent NVAF of $\geq 48 \mathrm{~h}$ or of unknown duration, who had been referred to our Echo Laboratory to perform a 2D-TEE examination for excluding the presence of spontaneous echo contrast (SEC) and/ or LAAT before scheduled ECV, between April 2016 and January 2020.

NVAF was defined according to the 2016 ESC Guidelines [15].

Main exclusion criteria were: atrial fibrillation (AF) duration $<48 \mathrm{~h}$, significant valvular heart disease (prosthetic valve, severe mitral regurgitation and more than mild mitral valve stenosis), hemodynamic instability, poor echocardiographic images unsuitable for LA size measurement by conventional 2D-TTE and for LA functional assessment by 2D-STE analysis, lack of consent.

The following patients' data were collected: age, gender, body surface area (BSA), prevalence of the most common cardiovascular risk factors (hypertension, smoking, diabetes mellitus, dyslipidemia), history of coronary artery disease and transient ischemic attack/stroke, and finally current medical treatment. In the case of vitamin $\mathrm{K}$ antagonists (VKAs), anticoagulation was considered therapeutic if there were at least three recent consecutive measurements of International Normalized Ratio (INR) showing values $\geq 2$.

The thromboembolic risk of each patient was assessed by the $\mathrm{CHA}_{2} \mathrm{DS}_{2}$-Vasc Risk Score [16].

All patients underwent medical history evaluation, physical examination, 12-lead electrocardiogram (ECG), conventional 2D-TTE implemented with 2D-STE analysis of LA strain and strain rate (SR) parameters and finally 2D-TEE. All examinations were performed in AF on the same day, before ECV if appropriate.

All procedures performed in the present study were in accordance with the ethical standards of the Institutional Research Committee and with the 1964 Helsinki Declaration and its later amendments or comparable ethical standards. A written informed consent was obtained from each participant included in the study and the protocol was approved by the local Ethics Committee.

\section{Transthoracic echocardiography}

All transthoracic echocardiographic examinations were performed by the same experienced cardiologist (A.S.), using commercially available Philips Sparq ultrasound 
machine (Philips Healthcare, Andover, Massachusetts, USA) with a $2.5 \mathrm{mHz}$ transducer. All images were acquired during AF. Five cardiac cycles were stored in cine-loop format for further offline analysis. Echocardiographic measurements were done for consecutive beats and thereafter averaged. All measurements were performed according to the criteria of the American Society of Echocardiography and the European Association of Cardiovascular Imaging [17-19]. The following echoDoppler parameters were collected: left ventricular mass index (LVMi) determined by the Devereux formula, left atrial volume index (LAVi) and left ventricular ejection fraction (LVEF) measured by the biplane modified Simpson's method, left ventricular (LV) diastolic function assessed by E/A ratio and average E/e' ratio, degree of concomitant mitral regurgitation (MR), tricuspid annular plane systolic excursion and finally systolic pulmonary artery pressure.

\section{Transesophageal echocardiography}

All transesophageal exams were performed by the same operator (A.S.) using Philips Sparq ultrasound machine (Philips Healthcare, Andover, Massachusetts, USA) with a $5 \mathrm{MHz}$ multiplane transducer.

The LAA was visualized from the mid-esophageal position at an angle (typically $45^{\circ}-90^{\circ}$ ) providing the longest apex to the orifice length. The grayscale frame rate was set to 60 to 90 frames/second. On average, five cardiac cycles were performed for both 2D and pulsed-wave (PW) Doppler recordings.

LAA flow measurements were obtained approximately 1 $\mathrm{cm}$ below the outlet of the LAA cavity using PW-Doppler with suitable gain and filter adjustments. The LAA emptying (LAA-EV) and the LAA filling velocities (LAA-FV) were recorded.

A thrombus in the LAA was defined an echo-dense mass of more than $2 \mathrm{~mm}$ in diameter attached to the LAA wall that could be distinguished from the surrounding endocardium or pectinate muscles [20].

SEC was diagnosed by the presence of characteristic dynamic smoke-like swirling echoes in the LA or the LAA, distinct from background white noise caused by excessive gain [21].

The intensity of SEC was graded according to the classification $(1$ to $4+)$ proposed by Fatkin et al [22]. Dense SEC was defined as grade $4+$.

All images were recorded on a hard disk for subsequent offline analysis.

\section{Speckle tracking echocardiography}

Two-dimensional STE was performed immediately after conventional TTE, during the same examination, by using the Philips QLAB 10.3.1 ultrasound software (Philips Healthcare, Andover, Massachusetts, USA). All acquired images were then analyzed offline by the same cardiologist (A.S.).

To calculate LA strain, we employed the same software currently used for the analysis of left ventricular (LV) function. The cardiac cycle was demarcated by indicating QRS onset. At first, we manually traced the atrial endocardium by using three reference points, the first one placed at the medial mitral annulus, the second one at the lateral mitral annulus and the third one at the atrial roof. The epicardial surface was automatically calculated, and after manually reducing the region of interest to the atrial thickness, to include only the atrial wall, the software automatically divided the atrial wall into seven segments. We repeated these steps from each of the two apical views: 4-chamber (seven segments) and 2-chamber (seven segments) using a "biplane method"; the apical 3-chamber view was excluded because the values of the antero-septal wall correspond to the ascending aorta.

Once the longitudinal atrial strain curves were obtained, the following measurement was performed: average peak positive global atrial strain (LA-GSA+), during the reservoir phase, plotted as a positive dotted curve from baseline to the peak positive of longitudinal strain (Fig. 1, panel a). Each dotted curve was automatically obtained from an average of the 7 atrial segments.

From the 2D atrial strain, SR curves were derived, which permitted the measurement of LA SR in AF: average peak positive LA SR (LA-GSR+), plotted as a positive dotted curve from baseline to the peak positive of longitudinal SR; average peak negative LA SR (LA-GSR-), plotted as a negative dotted curve from baseline to the peak negative of longitudinal SR; average peak-to-peak LA SR: the sum of the two peaks (Fig. 1, panel b).

To solve the problem of beat-to-beat variation in STE measurements we employed the index-beat method [23]. The STE results were estimated by using the ratio of preceding (RR1) to pre-preceding (RR2) RR interval. We selected the beat with the smallest difference between RR1-RR2 intervals.

\section{Statistical analysis}

Patients enrolled in the present study were categorized in two groups, i.e. patients with LAAT and those without. LAAT group included not only NVAF patients with a thrombus in the LAA but also those patients diagnosed with grade 4 SEC and/or severely depressed LAA-EV (< $25 \mathrm{~cm} / \mathrm{s}$ ) on 2D-TEE, because of the similar clinical characteristics of these patients. 


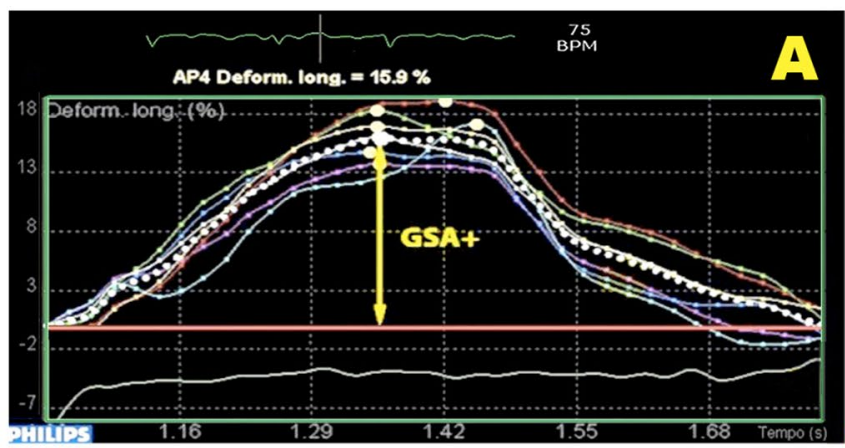

Fig. 1 Measurements of LA longitudinal strain (Panel a) and strain rate (Panel b) parameters by 2D-STE from the apical 4C-view in a NVAF patient enrolled in the present study. Panel A. Left atrial longitudinal strain parameters. Longitudinal strain curves of the seven atrial segments are depicted with different colors. The dotted line indicates the average atrial longitudinal strain. GSA+ (\%): positive global atrial strain (yellow arrow from the maximal positive peak of the dotted line to the red line). 2D, two dimensional. $L A$ left atrial,

For each group of patients, continuous data were summarized as mean \pm standard deviation (SD), while categorical data were presented as frequency and percentage.

Each continuous variable was checked through the Shapiro-Wilk test and all data were determined to be normally distributed. An independent two-tailed $t$ test was used to estimate the difference between the means. Categorical variables were compared using the chi-square test or the Fisher's exact test.

The primary end-point of the study was to identify the main conventional and functional echocardiographic parameters associated with LAAT on 2D-TEE in NVAF patients undergoing ECV.

Logistic regression analysis was performed to identify markers independently connected with LAAT. The covariates with $\mathrm{p}$ value $<0.05$ detected by univariate analysis were thereafter included in multivariate model. Risks were expressed as odds ratio (OR) with $95 \%$ confidence interval (CI).

We generated receiver operating characteristics (ROC) curves to determine the ability of different variables for discriminating between patients with LAAT and those without. The best cut-off for each parameter was obtained by maximizing the sum of sensitivity and specificity. Area under the curve (AUC) was then calculated.

The relationship between av. peak-to-peak LA SR and LAA flow velocities in the whole study population was evaluated by using the Pearson's correlation coefficient.

To evaluate intra- and interobserver variability in the assessment of main conventional and functional echocardiographic parameters, the key echocardiographic variables were finally re-measured in a sized subgroup of 15 patients (randomly selected) by the cardiologist who performed

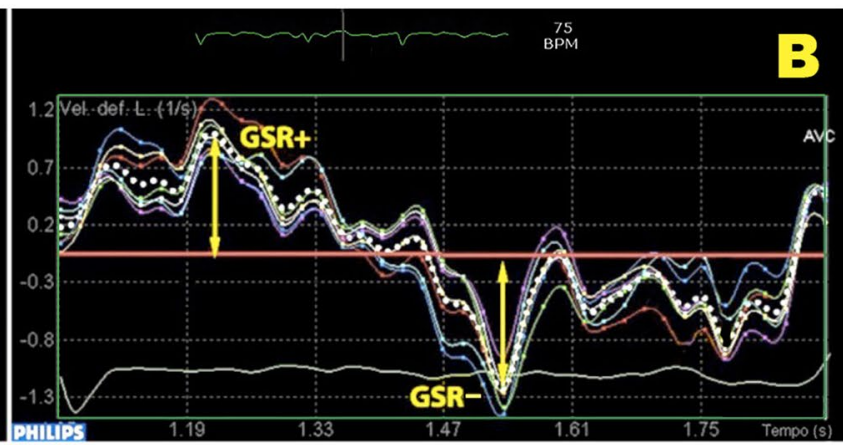

NVAF non-valvular atrial fibrillation, STE speckle tracking echocardiography. Panel B. Left atrial longitudinal SR parameters. The dotted line indicates the average SR. GSR+ (1/s), the first positive global strain rate, from the beginning of ventricular systole (yellow arrow from the maximal positive peak of the dotted line to the red line). GSR- (1/s), global early-diastolic strain rate (yellow arrow from the first maximal negative peak of the dotted line to the red line). $S R$ strain rate

all echocardiographic examinations and by a second one (M.L.). The analyses were performed in a blinded manner. Both raters chose the frame on which to perform each measurement. The intraclass correlation coefficient (ICC) was employed as statistical method for assessing intra- and interobserver measurement variability. An ICC of 0.70 or more was considered to indicate acceptable reliability.

Values of $\mathrm{p}<0.05$ were considered statistically significant.

Statistical analysis was performed with SPSS version 25 (SPSS Inc., Chicago, Illinois, USA).

\section{Results}

We retrospectively evaluated 125 consecutive NVAF patients ( $71.5 \pm 7.8$ yrs, 75 males) who underwent 2D-TEE before performing elective ECV.

A LAAT was detected in 35 patients (28\%), while the remaining 90 patients without LAAT (72\%) were classified as controls, according to previously established criteria.

\section{Demographic and clinical parameters}

Main demographic and clinical data for the study population and for both subgroups of patients are presented in Table 1 .

There were no significant differences between the two subgroups with respect to age, BSA, prevalence of most common cardiovascular risk factors, and the current medical treatment. Thromboembolic risk of LAAT group, as assessed by $\mathrm{CHA}_{2} \mathrm{DS}_{2}$-VASc Risk Score, was significantly higher than controls $(4.5 \pm 1.3$ vs $3.6 \pm 1.1, \mathrm{p}=0.0002)$. Moreover, all patients were treated with anticoagulants, 
Table 1 Main demographic and clinical characteristics of the whole study population and of two subgroups of patients, i.e. those with LAAT and those without

\begin{tabular}{lllll}
\hline Demographic and clinical parameters & $\begin{array}{l}\text { All NVAF } \\
\text { patients }(\mathrm{n}=\end{array}$ & $\begin{array}{l}\text { NVAF pts with } \\
\text { LAAT }(\mathrm{n}=35)\end{array}$ & $\begin{array}{l}\text { NVAF pts without } \\
\text { LAAT }(\mathrm{n}=90)\end{array}$ & p value \\
& $125)$ & & \\
\hline Age (yrs) & $71.5 \pm 7.8$ & $72.6 \pm 8.1$ & $71.0 \pm 7.7$ & 0.30 \\
Male sex (\%) & $75(60)$ & $19(54.3)$ & $56(62.2)$ & 0.42 \\
BSA (m $\left.{ }^{2}\right)$ & $1.91 \pm 0.2$ & $1.88 \pm 0.2$ & $1.93 \pm 0.2$ & 0.21 \\
Hypertension (\%) & $86(68.8)$ & $26(74.3)$ & $60(66.7)$ & 0.52 \\
Smokers (\%) & $42(33.6)$ & $12(34.3)$ & $30(33.3)$ & 0.91 \\
Type 2 diabetes (\%) & $31(24.8)$ & $11(31.4)$ & $20(22.2)$ & 0.18 \\
Dyslipidemia (\%) & $69(55.2)$ & $21(60)$ & $48(53.3)$ & 0.55 \\
History of CAD (\%) & $30(24)$ & $5(14.3)$ & $25(27.8)$ & 0.16 \\
Previous TIA/stroke (\%) & $22(17.6)$ & $12(34.3)$ & $10(11.1)$ & $\mathbf{0 . 0 0 4}$ \\
CHA ${ }_{2}$ DS ${ }_{2}$-Vasc Risk Score & $3.8 \pm 1.24$ & $4.5 \pm 1.3$ & $3.6 \pm 1.1$ & $\mathbf{0 . 0 0 0 2}$ \\
Heart rate (bpm) & $81.3 \pm 13.3$ & $83.4 \pm 13.5$ & $80.5 \pm 13.2$ & 0.27 \\
VKAs (\%) & $31(24.8)$ & $8(22.8)$ & $23(25.5)$ & 0.82 \\
Therapeutic anticoagulation $(\%)$ & $24(19.2)$ & $4(11.4)$ & $20(22.2)$ & 0.21 \\
NOACs (\%) & $94(75.2)$ & $26(74.3)$ & $68(75.5)$ & 0.88 \\
Antiplatelets (\%) & $23(18.4)$ & $4(11.4)$ & $19(21.1)$ & 0.30 \\
Antihypertensives (\%) & $98(78.4)$ & $28(80)$ & $70(77.8)$ & 0.98 \\
Beta blockers (\%) & $64(51.2)$ & $22(62.8)$ & $42(46.7)$ & 0.11 \\
Diuretics (\%) & $66(52.8)$ & $22(62.8)$ & $44(48.9)$ & 0.17 \\
Antiarrhythmics (\%) & $59(47.2)$ & $17(48.6)$ & $42(46.7)$ & 0.85 \\
\hline
\end{tabular}

LAAT left atrial appendage thrombosis, NOACs novel oral anticoagulants, NVAF non-valvular atrial fibrillation, SEC spontaneous echo contrast, TIA transient ischemic attack, VKAs vitamin $\mathrm{K}$ antagonists, Data are expressed as mean \pm SD or as number (percentage).

Significant $\mathrm{p}$ values are in bold. BSA, body surface area. $C A D$ coronary artery disease, $C H A_{2} D S_{2}-V A S c$ Congestive heart failure, Hypertension, Age at least 75 years (doubled), Diabetes, Stroke/transient ischemic attack/thromboembolism (doubled), Vascular disease (prior myocardial infarction, peripheral artery disease, or aortic plaque), Age 65-74 years, Sex category (female) mostly novel oral anticoagulants (NOACs) (75.2\%), without any statistically significant difference between the two subgroups.

\section{Conventional and functional transthoracic echocardiographic parameters}

Main conventional and functional echoDoppler parameters assessed by 2D-TTE implemented with 2D-STE analysis of LA strain and SR parameters are summarized in Table 2. Compared to controls, patients with LAAT had significantly lower LVEF $(46.4 \pm 14.5 \%$ vs $57.5 \pm$ $8.7 \%, \mathrm{p}<0.0001)$ and significantly higher LV filling pressures (LVFP), as measured by average E/e' ratio (18.4 \pm 5 vs $10.7 \pm 2.0, \mathrm{p}<0.0001)$. Both groups were comparable with regard to LV mass index (LVMi), LA volume index (LAVi) and the prevalence of moderate MR. All LA myocardial strain and SR parameters were adequately measured in all NVAF patients. Speckle-tracking analysis revealed significant differences between groups with and without LAAT. Notably, patients with LAAT had more pronounced impairment of all LA myocardial strain (i.e. LA-GSA+) and SR (i.e. LA-GSR+, LA-GSR- and peakto-peak LA SR) parameters (all $\mathrm{p}<0.0001$ ) in comparison to controls and to the accepted normal ranges [24, 25].

\section{Conventional transesophageal echocardiographic parameters}

Main conventional transesophageal echocardiographic parameters are reported in Table 3.

A grade 4 SEC was detected in 15 patients (12\%), while a thrombus in LAA was diagnosed in 20 patients (16\%).

Both LAA-EV $(23.9 \pm 3.9 \mathrm{~cm} / \mathrm{sec}$ vs $56.5 \pm 16.0 \mathrm{~cm} /$ sec, $\mathrm{p}<0.0001)$ and LAA-FV $(26.3 \pm 5.5 \mathrm{~cm} / \mathrm{sec}$ vs 54.0 $\pm 18.1 \mathrm{~cm} / \mathrm{sec}, \mathrm{p}<0.0001)$ were significantly lower in the LAAT group, while LAA size (as assessed by LAA ostial diameter and LAA 2D-area) was significantly greater in the LAAT group than controls. Notably, LAA cactus shape 
Table 2 Main conventional and functional echocardiographic parameters assessed by 2D-TTE in the whole study population and in the two subgroups of patients, i.e. those with LAAT and those without
Table 3 Main conventional echocardiographic parameters assessed by 2D-TEE in the whole study population and in the two subgroups of patients, i.e. those with LAAT and those without

\begin{tabular}{llllc}
\hline $\begin{array}{l}\text { Conventional and functional } \\
\text { TTE parameters }\end{array}$ & $\begin{array}{l}\text { All NVAF } \\
\text { patients }(\mathrm{n}= \\
125)\end{array}$ & $\begin{array}{l}\text { NVAF pts with } \\
\text { LAAT }(\mathrm{n}=35)\end{array}$ & $\begin{array}{l}\text { NVAF pts without } \\
\text { LAAT }(\mathrm{n}=90)\end{array}$ & p value \\
\hline LVEDVi $\left(\mathrm{ml} / \mathrm{m}^{2}\right)$ & $42.2 \pm 14.2$ & $40.0 \pm 13.5$ & $43.0 \pm 14.4$ & 0.29 \\
RWT & $0.41 \pm 0.1$ & $0.42 \pm 0.1$ & $0.41 \pm 0.1$ & 0.61 \\
LVMi $\left(\mathrm{g} / \mathrm{m}^{2}\right)$ & $101.9 \pm 29.3$ & $103.6 \pm 28.9$ & $101.3 \pm 29.6$ & 0.69 \\
LVEF $(\%)$ & $54.4 \pm 11.7$ & $46.4 \pm 14.5$ & $57.5 \pm 8.7$ & $<\mathbf{0 . 0 0 0 1}$ \\
Average E/e' ratio & $12.8 \pm 4.7$ & $18.4 \pm 5.0$ & $10.7 \pm 2.0$ & $<\mathbf{0 . 0 0 0 1}$ \\
LAVi (ml/m $\left.{ }^{2}\right)$ & $46.9 \pm 10.6$ & $48.4 \pm 8.3$ & $46.4 \pm 11.3$ & 0.34 \\
Av. LA-GSA + $(\%)$ & $13.1 \pm 4.8$ & $9.0 \pm 2.5$ & $14.8 \pm 4.5$ & $<\mathbf{0 . 0 0 0 1}$ \\
Av. Peak positive LA SR $\left(\mathrm{s}^{-1}\right)$ & $1.65 \pm 0.49$ & $1.36 \pm 0.21$ & $1.78 \pm 0.52$ & $<\mathbf{0 . 0 0 0 1}$ \\
Av. Peak negative LA SR $\left(\mathrm{s}^{-1}\right)$ & $0.90 \pm 0.23$ & $0.76 \pm 0.24$ & $0.96 \pm 0.20$ & $<\mathbf{0 . 0 0 0 1}$ \\
Av. Peak to peak LA SR $\left(\mathrm{s}^{-1}\right)$ & $2.54 \pm 0.58$ & $2.12 \pm 0.31$ & $2.70 \pm 0.57$ & $<\mathbf{0 . 0 0 0 1}$ \\
Mild MR $(\%)$ & $95(76)$ & $30(85.7)$ & $65(72.2)$ & 0.16 \\
Moderate MR $(\%)$ & $30(24)$ & $5(14.3)$ & $25(27.8)$ & 0.16 \\
RVIT (mm) & $33.5 \pm 5.4$ & $34.5 \pm 5.2$ & $33.1 \pm 5.5$ & 0.20 \\
TAPSE (mm) & $18.2 \pm 4.3$ & $17.5 \pm 4.1$ & $18.5 \pm 4.4$ & 0.25 \\
SPAP (mmHg) & $37.7 \pm 11.5$ & $39.3 \pm 11.2$ & $37.0 \pm 11.7$ & 0.32 \\
\hline
\end{tabular}

Significant $\mathrm{p}$ values are in bold. 2D, two-dimensional

$A v$ average, $G S A+$ peak positive global atrial strain, $L A$ left atrial, $L A V i$ left atrial volume indexed, $L A A T$ left atrial appendage thrombosis, $L V E D V i$ left ventricular end-diastolic volume index, $L V E F$ left ventricular ejection fraction, $L V M i$ left ventricular mass indexed, $M R$ mitral regurgitation, $N V A F$ non-valvular atrial fibrillation, RVIT right ventricular inflow tract, $R W T$ relative wall thickness, $S E C$ spontaneous echo contrast, $S P A P$ systolic pulmonary artery pressure, $S R$ strain rate, $S T E$ speckle tracking echocardiography, TAPSE tricuspid annular plane systolic excursion, TTE transthoracic echocardiography. Data are expressed as mean $\pm \mathrm{SD}$ or as number (percentage)

\begin{tabular}{lllll}
\hline Conventional TEE parameters & $\begin{array}{l}\text { All NVAF } \\
\text { patients }(\mathrm{n}= \\
125)\end{array}$ & $\begin{array}{l}\text { NVAF pts with } \\
\text { LAAT }(\mathrm{n}=35)\end{array}$ & $\begin{array}{l}\text { NVAF pts without } \\
\text { LAAT }(\mathrm{n}=90)\end{array}$ & p value \\
& $15(12)$ & $15(43)$ & - & - \\
\hline Presence of grade 4 SEC $(\%)$ & $20(57)$ & - & - \\
Presence of LAA thrombus $(\%)$ & $20(16)$ & $2(5.7)$ & $40(44.4)$ & $<\mathbf{0 . 0 0 0 1}$ \\
LAA CW shape $(\%)$ & $42(33.6)$ & $5(14.3)$ & $25(27.8)$ & 0.16 \\
LAA WS shape $(\%)$ & $30(24)$ & $10(28.6)$ & $15(16.7)$ & 0.14 \\
LAA CF shape $(\%)$ & $25(20)$ & $18(51.4)$ & $10(11.1)$ & $<\mathbf{0 . 0 0 0 1}$ \\
LAA Cactus shape $(\%)$ & $28(22.4)$ & $2.1 \pm 0.3$ & $1.9 \pm 0.3$ & $\mathbf{0 . 0 0 1}$ \\
LAA ostial diameter $(\mathrm{mm})$ & $2.0 \pm 0.3$ & $6.5 \pm 2.3$ & $5.1 \pm 1.6$ & $\mathbf{0 . 0 0 0 2}$ \\
LAA 2D-area $\left(\mathrm{cm}^{2}\right)$ & $5.5 \pm 1.9$ & $23.9 \pm 3.9$ & $56.5 \pm 16.0$ & $<\mathbf{0 . 0 0 0 1}$ \\
LAA-EV $(\mathrm{cm} / \mathrm{s})$ & $47.3 \pm 20.1$ & $26.3 \pm 5.5$ & $54.0 \pm 18.1$ & $<\mathbf{0 . 0 0 0 1}$ \\
LAA- FV $(\mathrm{cm} / \mathrm{s})$ & $46.2 \pm 20.0$ & &
\end{tabular}

Significant $\mathrm{p}$ values are in bold. 2D, two-dimensional

$C F$ cauliflower, $C W$ chicken wing, $E V$ emptying velocity, $F V$ filling velocity, $L A A$ left atrial appendage, $L A A T$ left atrial appendage thrombosis, $N V A F$ non-valvular atrial fibrillation, $S E C$ spontaneous echo contrast, TEE transesophageal echocardiography, WS windsock. Data are expressed as mean \pm SD or as number (percentage).
(51.4\%) was the most common LAA shape associated to LAAT. Conversely, LAA chicken wing shape (44.4\%) was prevalent in controls.
Figure 2 depicts an example of LA-GSA+ assessed by 2D-STE analysis, in a NVAF patient with LAAT enrolled in the study. 
Fig. 2 Example of LA-GSA+ assessed by 2D-STE analysis, in a NVAF patient with LAAT enrolled in the present study. Yellow arrow indicates the LA-GSA+ value measured by 2D-STE analysis. This value $(5.6 \%)$ was significantly depressed in comparison to controls and to the accepted normal ranges. $2 D$ two-dimensional, $G S A+$ peak positive global atrial strain, $L A$ left atrial, $N V A F$ non-valvular atrial fibrillation, STE speckle tracking echocardiography

Table 4 The factors associated with LAAT in univariate and multivariate logistic regression analysis

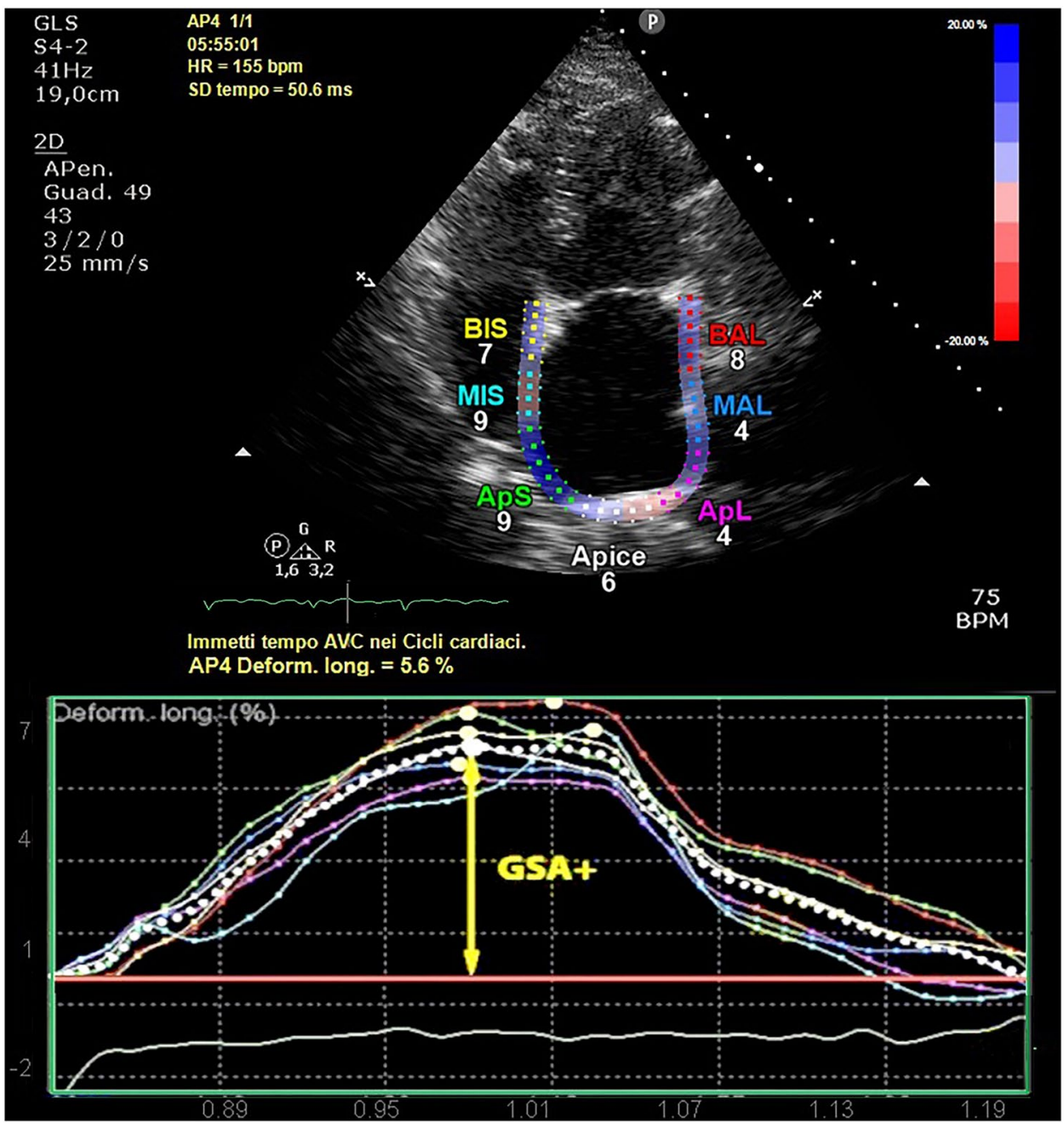

\begin{tabular}{|c|c|c|c|c|c|c|}
\hline \multirow[b]{2}{*}{ Variables } & \multicolumn{3}{|c|}{ Univariate logistic regression analysis } & \multicolumn{3}{|c|}{$\begin{array}{l}\text { Multivariate logistic regression } \\
\text { analysis }\end{array}$} \\
\hline & OR & $95 \% \mathrm{CI}$ & $\mathrm{p}$ value & OR & $95 \% \mathrm{CI}$ & $\mathrm{p}$ value \\
\hline $\mathrm{CHA}_{2} \mathrm{DS}_{2}$-Vasc Risk Score & 1.92 & $1.33-2.77$ & $<0.0001$ & 1.26 & $0.53-2.98$ & 0.59 \\
\hline $\operatorname{LVMi}\left(\mathrm{g} / \mathrm{m}^{2}\right)$ & 1.00 & $0.99-1.01$ & 0.68 & & & \\
\hline LVEF (\%) & 0.92 & $0.88-0.96$ & $<0.0001$ & 0.88 & $0.81-0.97$ & 0.01 \\
\hline Average E/e' ratio & 2.25 & $1.64-3.10$ & $<0.0001$ & 2.36 & $1.41-3.98$ & 0.001 \\
\hline $\operatorname{LAVi}\left(\mathrm{ml} / \mathrm{m}^{2}\right)$ & 1.02 & $0.98-1.05$ & 0.35 & & & \\
\hline Average LA-GSA+ $(\%)$ & 0.59 & $0.47-0.74$ & $<0.0001$ & 0.57 & $0.36-0.90$ & 0.01 \\
\hline Moderate MR & 0.43 & $0.15-1.24$ & 0.12 & & & \\
\hline
\end{tabular}

Significant $\mathrm{p}$ values are highlighted in bold

$\mathrm{CHA}_{2} \mathrm{DS}_{2}$-VASc Congestive heart failure, Hypertension, Age at least 75 years (doubled), Diabetes, Stroke/ transient ischemic attack/thromboembolism (doubled), Vascular disease (prior myocardial infarction, peripheral artery disease, or aortic plaque), Age 65-74 years, Sex category (female). Av average. $C I$ confidence interval, $L A$ left atrial, GSA+ peak positive global atrial strain, $L A A T$ left atrial appendage thrombosis, $L A V i$ left atrial volume index, $L V E F$ left ventricular ejection fraction, $L V M i$ left ventricular mass index, $M R$ mitral regurgitation, $O R$ odds ratio 
Table 5 ROC curves for the identifying of LAAT. 2D, two-dimensional. AUC, area under the curve

ROC curves for the identifying of LAAT

\begin{tabular}{|c|c|c|c|c|c|c|c|}
\hline VARIABLES & AUC & $\mathrm{p}$ value & $\begin{array}{l}\text { Criterion of highest } \\
\text { diagnostic value }\end{array}$ & Sensitivity (\%) & $\begin{array}{l}\text { Specificity } \\
(\%)\end{array}$ & PPV (\%) & NPV (\%) \\
\hline $\mathrm{CHA}_{2} \mathrm{DS}_{2}$-VASc Risk Score & 0.71 & $<0.0001$ & $\geq 4$ & 83 & 50 & 62 & 75 \\
\hline $\operatorname{LVEF}(\%)$ & 0.73 & $<0.0001$ & $\leq 45$ & 49 & 89 & 82 & 63 \\
\hline Average E/e' ratio & 0.93 & $<0.0001$ & $\geq 15$ & 77 & 89 & 87 & 79 \\
\hline Average LA-GSA+ (\%) & 0.95 & $<0.0001$ & $\leq 9.1$ & 100 & 87 & 88 & 100 \\
\hline
\end{tabular}

$\mathrm{CHA}_{2} \mathrm{DS}_{2}$-VASc Congestive heart failure, Hypertension, Age at least 75 years (doubled), Diabetes, Stroke/transient ischemic attack/thromboembolism (doubled), Vascular disease (prior myocardial infarction, peripheral artery disease, or aortic plaque), Age 65-74 years, Sex category (female). $L A$ left atrial, $G S A+$ peak positive global atrial strain, $L V E F$ left ventricular ejection fraction, $N P V$ negative predictive value, $P P V$ predictive positive value, $R O C$ receiver operating characteristics.

\section{Factors associated with LAAT presence}

Table 4 lists univariate and multivariate parameters associated with LAAT. Multivariate logistic regression analysis revealed that, differently from $\mathrm{CHA}_{2} \mathrm{DS}_{2}$-Vasc Risk Score, the following variables were independently associated with the presence of LAAT on 2D-TEE in NVAF patients: LVEF (OR $0.88,95 \% \mathrm{CI} 0.81-0.97, \mathrm{p}=0.01$ ), average E/e' ratio (OR 2.36, 95\% CI 1.41-3.98, $\mathrm{p}=0.001$ ), and LA-GSA+ (OR $0.57,95 \%$ CI $0.36-0.90, \mathrm{p}=0.01$ ).

Moreover, we conducted ROC curve analysis to evaluate the diagnostic value of LVEF, average E/e' ratio and LAGSA+ for LAAT prediction (Table 5).

All parameters had good AUC, ranging from 0.71 (for $\mathrm{CHA}_{2} \mathrm{DS}_{2}$-Vasc Risk Score) to 0.95 (for LA-GSA+). Cutoff values for LVEF, average E/e' and LA-GSA+ for the presence of LAAT were $\leq 45 \%, \geq 15$ and $\leq 9.1 \%$, respectively (all $\mathrm{p}<0.0001$ ). The highest diagnostic performance was noted for LA-GSA+ (optimal cut-off $\leq 9.1 \%$, AUC 0.95 , sensitivity $100 \%$, specificity $87 \%$, positive predictive value $88 \%$, negative predictive value $100 \%$ ) whereas the lowest performance was noted for $\mathrm{CHA}_{2} \mathrm{DS}_{2}$-Vasc Risk
Score (optimal cut-off $\geq 4$, AUC 0.71 , sensitivity $83 \%$, specificity $50 \%$ ).

Finally, we observed a strong positive correlation of av. peak-to-peak LA SR with both LAA-FV $(r=0.86)$ and LAA-EV $(r=0.83)$ in the whole study population (Fig. 3, Panels a and $b$, respectively).

Figure 4 depicts examples of grade 4 SEC (Panel a) and thrombus (Panel b) in the LAA detected by 2D-TEE examination in two NVAF patients enrolled in the present study.

\section{Intra- and inter-observer variability}

A detailed intra- and interobserver variability analysis of the main conventional and functional echocardiographic parameters, re-measured in a group of 15 randomly selected NVAF patients, is reported in the Supplemental Table. Intra- and interobserver agreement between the raters, expressed as ICCs with $95 \% \mathrm{CIs}$, ranged from 0.78 to 0.91 and from 0.76 to 0.87 , respectively.
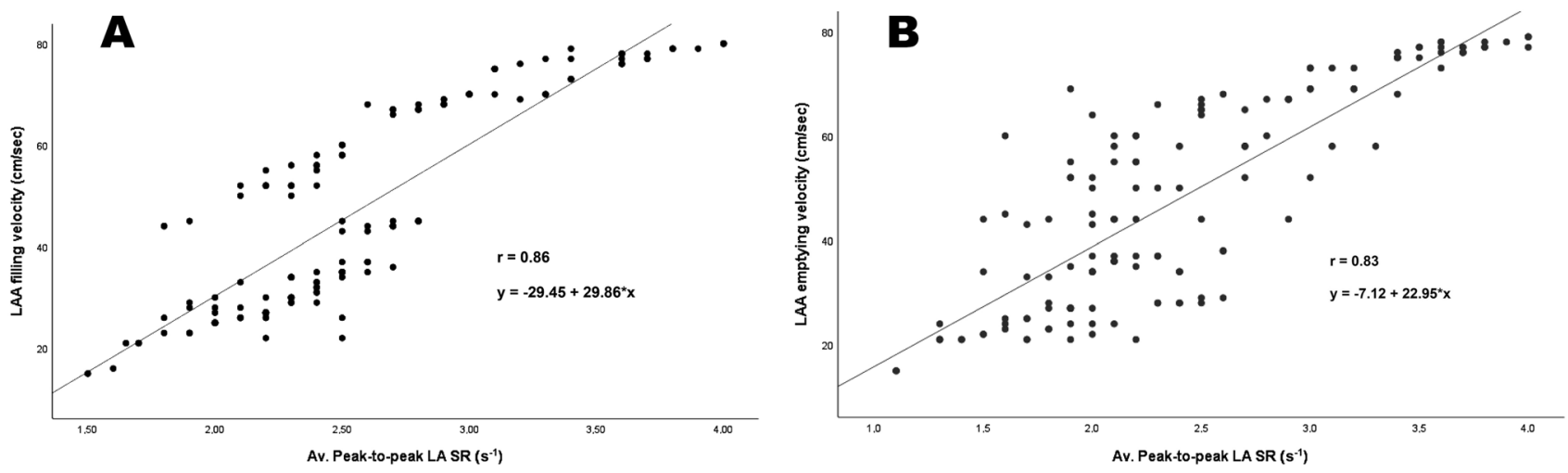

Fig. 3 The correlation of peak-to-peak LA SR with LAA-FV (Panel a) and LAA-EV (Panel b) in the whole study population, evaluated by using the Pearson's correlation coefficient. $E V$ emptying velocity, $F V$ filling velocity, $L A$ left atrial, $L A A$ left atrial appendage, $S R$ strain rate 

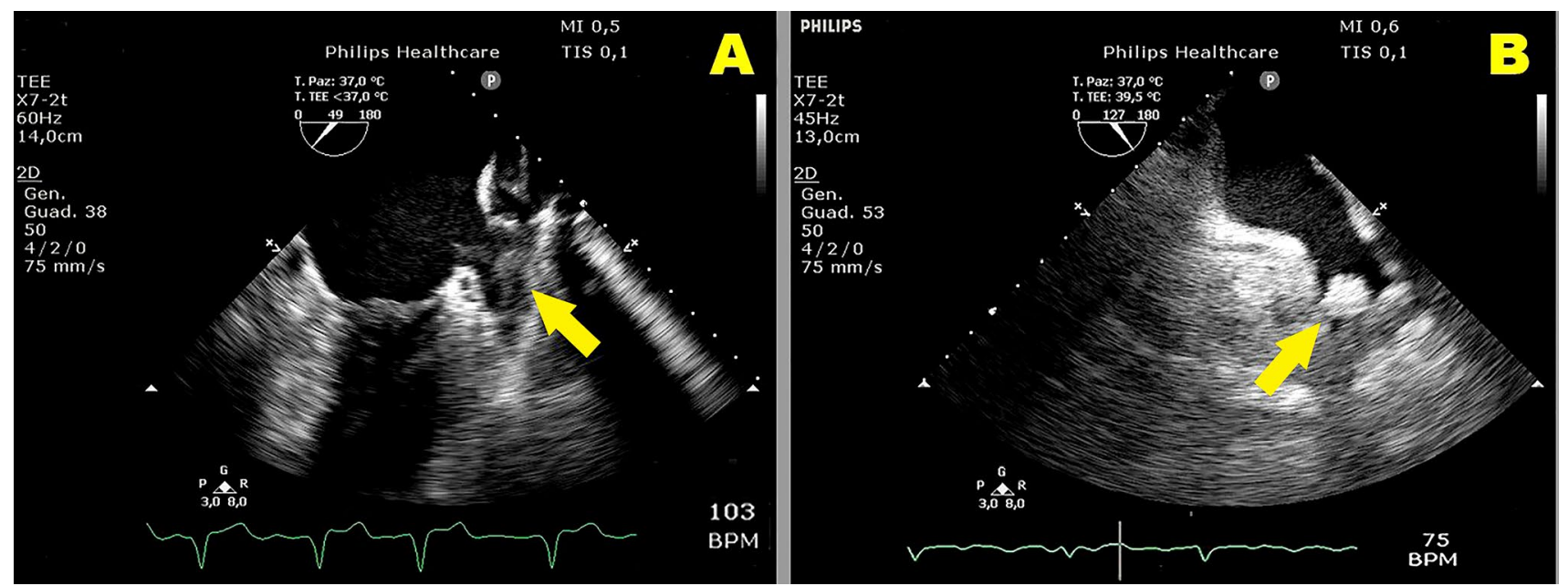

Fig. 4 Examples of grade 4 SEC (Panel a, yellow arrow) and thrombus (Panel b, yellow arrow) in the LAA detected by 2D-TEE examination in two NVAF patients enrolled in the present study

\section{Discussion}

The primary mechanism of thrombus formation in LA and LAA is their dysfunction in NVAF patients [26]. In the case of LA/LAA dysfunction, prethrombotic phenomena, such as sludge and SEC, and/or thrombi may develop in the LAA due to reduced blood flow and stasis [27, 28]. All these factors have been associated with a worse outcome [29-33]. In patients with persistent NVAF, higher left atrial dyssynchrony and compromised LA contraction and relaxation may contribute to the occurrence of low flow velocities in the LAA [29, 31]. Notably, reduced LAA blood flow velocity below $25 \mathrm{~cm} / \mathrm{s}$ has been shown to increase the risk of developing blood clots [4, 29, 31]. LAA segmental anatomy is another factor that increases the likelihood of thrombi [34-36]. In particular, a nonchicken wing LAA morphology [37] and a multi-lobar LAA [38] may favor blood stasis and precipitate thrombus formation.

All these parameters are usually detected during TEE [4]. However, the performance of this invasive examination carries a heightened risk for transmission of severe acute respiratory syndrome coronavirus 2 (SARS-CoV-2), especially in nonintubated patients, secondary to the aerosolization of a large amount of virus due to coughing or gagging. Therefore, during the current COVID-19 pandemic, TEE should be postponed or canceled and alternative imaging modalities considered $[5,6]$. The use of contrast enhanced computed tomography (CT) and magnetic resonance imaging (MRI) has emerged as an alternative to TEE for exclusion of LAAT prior to cardioversion [39]. Main limitations of these procedures are: variable waiting lists and waiting times among different Institutions, risk of transporting a patient through the hospital to the computed tomographic or MRI scanner, need to disinfect the CT or MRI room, use of iodinated contrast and radiation for $\mathrm{CT}$ and finally long scan times for MRI [6].

On the other hand, the potential role of conventional TTE in the risk stratification of NVAF patients scheduled for ECV is actually overlooked [4]. It's known that AF has been associated with LA electromechanical remodeling $[15,40]$. TTE is a useful diagnostic tool to examine the LA structural remodeling which occurs in NVAF patients [41-43]. This structural remodeling is characterized by progressive LA enlargement and increased atrial stiffness [44, 45], especially in cases with long AF duration [46].

LA reservoir strain measured by STE analysis is a marker of the structural atrial remodeling that is intrinsic to the pathophysiology of the AF and represents the substrate for its maintenance [41, 47].

Previous studies demonstrated a negative correlation between LA strain and the extent of fibrosis on delayedenhancement cardiac magnetic resonance in patients with persistent NVAF [48, 49]. Moreover, a severely impaired LA strain, reflecting decreased LAA flow velocities measured at TEE, may contribute to the increased pro-thrombotic profile of NVAF patients with compromised LA mechanical function [50].

To the best of our knowledge, there are only a few studies in the literature that have directly evaluated LA myocardial strain and SR parameters with 2D-STE analysis in patients with persistent NVAF [11-14].

Many of these parameters show a wide variability in NVAF patients, mainly due to the presence of the arrhythmia at the time of performing the ultrasound examination. In addition, analysis of systolic deformation in patients with $\mathrm{AF}$ is rather difficult because the magnitude of strain is very small and requires extreme precision in the assessment of STE-derived images. 
In the present study, we performed a retrospective crosssectional analysis of $125 \mathrm{NVAF}$ patients scheduled for ECV, in order to investigate the association between main conventional and functional echocardiographic parameters and the presence of LAAT on 2D-TEE. All patients underwent a complete conventional transthoracic examination implemented with 2D-STE analysis of LA myocardial strain and SR parameters in AF.

Despite the thinness of the LA walls, a complete LA morphological and functional assessment was possible in all the patients enrolled.

The 2D-LA strain and SR values obtained in NVAF patients with LAAT were significantly impaired in comparison to controls and to the accepted normal ranges [24, 25].

Our results revealed that several factors, only partially related to each other, contributed to LAAT formation in NVAF: LV haemodynamics (LV systolic and diastolic function), the structural remodeling of the atrial muscle tissues, haemoreological factors and finally LAA morphology.

At univariate logistic regression analysis, the main parameters independently associated with LAAT were the following: a clinical parameter $\left(\mathrm{CHA}_{2} \mathrm{DS}_{2}\right.$-Vasc Risk Score), two conventional echocardiographic parameters (LVEF and average E/e' ratio) and finally a functional echocardiographic parameter (LA-GSA+). Interestingly, at multivariate logistic regression analysis, the clinical parameter lost its statistical significance, while the three echocardiographic parameters retained a statistical significance. In ROC curve analysis, LA-GSA+ showed the highest diagnostic performance.

Additionally, our TEE-derived data suggest that haemoreological factors involved in the LA-LAA complex may play a relevant role for LAAT occurrence: both PW-Doppler derived LAA-FV and LAA-EV were significantly reduced in NVAF patients with LAAT. Moreover, our results demonstrated a strong linear correlation of LA peak-to-peak SR with LAA flow velocities. These findings are expression of a robust mechanical concordance between LA and LAA in NVAF patients.

Main clinical implication of our findings is that NVAF patients with LA-GSA $+\leq 9.1 \%$ should be considered to have a significantly increased probability of LAAT on 2D-TEE before scheduled ECV. On the other hand, NVAF patients with LA-GSA $+>9.1 \%$ should be considered to have a significantly lower probability of LAAT. Therefore, these two groups of NVAF patients could be reasonably spared from 2D-TEE examination.

Consistent with previous studies [11-14], the present study demonstrated that 2D-STE is a feasible method for the evaluation of regional LA mechanics in NVAF patients. However, several limitations of the present study should be addressed. The main limitation could be that all conventional and functional echocardiographic parameters were acquired during AF. The presence of the arrhythmia could partly affect the validity of some of the parameters considered, due to an increased beat-to-beat variability. Nevertheless, all LA myocardial strain and SR parameters were adequately recorded in the whole study population by using the indexbeat method [23]. Secondly, in our series of patients it was not possible to use the integrated backscatter technique to quantify SEC [51, 52]. On the other hand, it can be assumed that quantitative classification of SEC would not add any relevant information, since only severe SEC was used as inclusion criterion for LAAT group.

Third, in our series the prevalence of LAAT was $28 \%$, a high value compared to those reported in the literature [53]. A possible explanation of this finding could be that, in our study, even NVAF patients with high-grade SEC were considered as patients with LAAT. From the point of view of clinical behavior, these patients should be managed as patients with LAAT. In fact, the high-grade SEC within LA/ LAA, that is related to the initial formation of fibrin bridges between red blood cells, should be considered a pre-thrombotic condition, and its echocardiographic detection is an absolute contraindication to perform cardioversion.

Another reason for the high prevalence of LAAT in our study population was that also patients who were taking anticoagulant therapy only for a short time period were included in the study. A re-evaluation after three weeks or more of adequate anticoagulation therapy could have shown disappearance of LAAT in these patients.

Finally, due to the cross-sectional nature of the study, we were not able to accurately determine the value of assessing global and regional LA mechanics for the prediction of future thromboembolic events. Therefore, these results need to be validated in a larger, prospective study.

\section{Conclusions}

2D-STE analysis of LA mechanics is technically feasible and is able to provide incremental information over the $\mathrm{CHA}_{2} \mathrm{DS}_{2}$-Vasc Risk Score for discriminating between NVAF patients with and without LAAT.

LA myocardial strain parameters are significantly correlated with the dynamics of blood flow in the LA-LAA complex.

Although its use in daily practice is limited, a complete 2D-STE analysis of LA myocardial strain and SR parameters may improve thrombotic risk assessment of NVAF patients scheduled for ECV and may contribute to spare from 2D-TEE examination those patients with significantly increased or significantly decreased LAAT probability.

Acknowledgements This work has been supported by Italian Ministry of Health Ricerca Corrente - IRCCS MultiMedica. 


\section{Compliance with ethical standards}

Conflict of interest We wish to confirm that there are no conflicts of interest associated with this publication. Andrea Sonaglioni declares that he has no conflict of interest. Michele Lombardo declares that he has no conflict of interest. Gian Luigi Nicolosi declares that he has no conflict of interest. Elisabetta Rigamonti declares that she has no conflicts of interest. Claudio Anzà declares that he has no conflicts of interest.

Ethical approval All procedures performed in the present study were in accordance with the ethical standards of the institutional research committee and with the 1964 Helsinki declaration and its later amendments or comparable ethical standards.

Informed consent Informed consent was obtained from all individual participants included in the study.

\section{References}

1. Wolf PA, Dawber TR, Thomas HE Jr, Kannel WB (1978) Epidemiologic assessment of chronic atrial fibrillation and risk of stroke: the Framingham study. Neurology 28:973-977

2. Benjamin EJ, Wolf PA, D'Agostino RB, Silbershatz H, Kannel WB, Levy D (1998) Impact of atrial fibrillation on the risk of death: the Framingham Heart Study. Circulation 98:946-952

3. Blackshear JL, Odell JA (1996) Appendage obliteration to reduce stroke in cardiac surgical patients with atrial fibrillation. Ann Thorac Surg 61:755-759

4. Providência R, Trigo J, Paiva L, Barra S (2013) The role of echocardiography in thromboembolic risk assessment of patients with nonvalvular atrial fibrillation. J Am Soc Echocardiogr 26:801-812

5. Skulstad H, Cosyns B, Popescu BA et al (2020) COVID-19 pandemic and cardiac imaging: EACVI recommendations on precautions, indications, prioritization, and protection for patients and healthcare personnel. Eur Heart J Cardiovasc Imaging 21:592-598

6. Kirkpatrick JN, Mitchell C, Taub C, Kort S, Hung J, Swaminathan M (2020) ASE Statement on Protection of Patients and Echocardiography Service Providers During the 2019 Novel Coronavirus Outbreak: Endorsed by the American College of Cardiology. J Am Soc Echocardiogr 33:648-653

7. Vianna-Pinton R, Moreno CA, Baxter CM, Lee KS, Tsang TS, Appleton CP (2009) Two-dimensional speckle-tracking echocardiography of the left atrium: feasibility and regional contraction and relaxation differences in normal subjects. J Am Soc Echocardiogr 22:299-305

8. Cameli M, Lisi M, Focardi M et al (2012) Left atrial deformation analysis by speckle tracking echocardiography for prediction of cardiovascular outcomes. Am J Cardiol 110:264-9

9. Sonaglioni A, Vincenti A, Baravelli M et al (2019) Prognostic value of global left atrial peak strain in patients with acute ischemic stroke and no evidence of atrial fibrillation. Int J Cardiovasc Imaging 35:603-613

10. Sonaglioni A, Caminati A, Lipsi R et al (2020) Early left atrial dysfunction in idiopathic pulmonary fibrosis patients without chronic right heart failure. Int J Cardiovasc Imaging 36:1711-1723

11. Providência R, Faustino A, Paiva L et al (2012) Cardioversion safety in patients with nonvalvular atrial fibrillation: which patients can be spared transesophageal echocardiography? Blood Coagul Fibrinolysis 23:597-602

12. Providência R, Faustino A, Ferreira MJ et al (2013) Evaluation of left atrial deformation to predict left atrial stasis in patients with non-valvular atrial fibrillation - a pilot-study. Cardiovasc Ultrasound 11:44

13. Obokata M, Negishi K, Kurosawa K et al (2014) Left atrial strain provides incremental value for embolism risk stratification over $\mathrm{CHA}_{2} \mathrm{DS}_{2}$-VASc score and indicates prognostic impact in patients with atrial fibrillation. J Am Soc Echocardiogr 27:709-716.e4

14. Kupczynska K, Michalski BW, Miskowiec D et al (2017) Association between left atrial function assessed by speckle-tracking echocardiography and the presence of left atrial appendage thrombus in patients with atrial fibrillation. Anatol J Cardiol 18:15-22

15. Kirchhof P, Benussi S, Kotecha D et al (2016) 2016 ESC Guidelines for the management of atrial fibrillation developed in collaboration with EACTS. Eur Heart J 37:2893-2962

16. Lip GY, Nieuwlaat R, Pisters R, Lane DA, Crijns HJ (2010) Refining clinical risk stratification for predicting stroke and thromboembolism in atrial fibrillation using a novel risk factorbased approach: the euro heart survey on atrial fibrillation. Chest 137:263-272

17. Lang RM, Badano LP, Mor-Avi V et al (2015) Recommendations for cardiac chamber quantification by echocardiography in adults: an update from the American Society of Echocardiography and the European Association of Cardiovascular Imaging. J Am Soc Echocardiogr 28:1-39.e14

18. Nagueh SF, Smiseth OA, Appleton CP et al (2016) Recommendations for the Evaluation of Left Ventricular Diastolic Function by Echocardiography: An Update from the American Society of Echocardiography and the European Association of Cardiovascular Imaging. J Am Soc Echocardiogr 29:277-314

19. Rudski LG, Lai WW, Afilalo J et al (2010) Guidelines for the echocardiographic assessment of the right heart in adults: a report from the American Society of Echocardiography endorsed by the European Association of Echocardiography, a registered branch of the European Society of Cardiology, and the Canadian Society of Echocardiography. J Am Soc Echocardiogr 23:685-788

20. Pepi M, Evangelista A, Nihoyannopoulos P et al (2010) Recommendations for echocardiography use in the diagnosis and management of cardiac sources of embolism: European Association of Echocardiography (EAE) (a registered branch of the ESC). Eur J Echocardiogr 11:461-76

21. Beppu S, NimuraY SakakibaraH, NagataS ParkY D, IzumiS, (1985) Smoke-like echo in the left atrial cavity in mitral valve disease: its features and significance. J. Am. Coll. Cardiol 6:744-9

22. Fatkin D, KellyR P, FeneleyM P (1994) Relations between left atrial appendage blood flow velocity, spontaneous echocardiographic contrast and thromboembolic risk in vivo. J. Am. Coll. Cardiol 23:961-9

23. Kusunose K, Yamada H, Nishio S et al (2012) Index-beat assessment of left ventricular systolic and diastolic function during atrial fibrillation using myocardial strain and strain rate. J Am Soc Echocardiogr 25:953-9

24. Miglioranza MH, Badano LP, Mihăilă S et al (2016) Physiologic Determinants of Left Atrial Longitudinal Strain: A TwoDimensional Speckle-Tracking and Three-Dimensional Echocardiographic Study in Healthy Volunteers. J Am Soc Echocardiogr 29:1023-1034.e3

25. Pathan F, D'Elia N, Nolan MT, Marwick TH, Negishi K (2017) Normal Ranges of Left Atrial Strain by Speckle-Tracking Echocardiography: A Systematic Review and Meta-Analysis. J Am Soc Echocardiogr 30:59-70.e8

26. Alessandri N, Mariani S, Ciccaglioni A et al (2003) Thrombus formation in the left atrial appendage in the course of atrial fibrillation. Eur Rev Med Pharmacol Sci 7:65-73

27. Al-Saady NM, Obel OA, Camm AJ (1999) Left atrial appendage: structure, function, and role in thromboembolism. Heart $82: 547-554$ 
28. Wolf PA, Abbott RD, Kannel WB (1991) Atrial fibrillation as an independent risk factor for stroke: the Framingham Study. Stroke 22:983-988

29. Troughton RW, Asher CR, Klein AL (2003) The role of echocardiography in atrial fibrillation and cardioversion. Heart 89:1447-1454

30. Lowe BS, Kusunose K, Motoki H et al (2014) Prognostic significance of left atrial appendage "sludge" in patients with atrial fibrillation: a new transesophageal echocardiographic thromboembolic risk factor. J Am Soc Echocardiogr 27:1176-1183

31. Zabalgoitia M, Halperin JL, Pearce LA, Blackshear JL, Asinger RW, Hart RG (1998) Transesophageal echocardiographic correlates of clinical risk of thromboembolism in nonvalvular atrial fibrillation. Stroke Prevention in Atrial Fibrillation III Investigators. J Am Coll Cardiol 31:1622-1626

32. Leung DY, Black IW, Cranney GB, Hopkins AP, Walsh WF (1994) Prognostic implications of left atrial spontaneous echo contrast in nonvalvular atrial fibrillation. J Am Coll Cardiol 24:755-762

33. Bernhardt P, Schmidt H, Hammerstingl C, Lüderitz B, Omran $\mathrm{H}$ (2005) Patients with atrial fibrillation and dense spontaneous echo contrast at high risk a prospective and serial follow-up over 12 months with transesophageal echocardiography and cerebral magnetic resonance imaging. J Am Coll Cardiol 45:1807-1812

34. Meissner I, Whisnant JP, Khandheria BK et al (1999) Prevalence of potential risk factors for stroke assessed by transesophageal echocardiography and carotid ultrasonography: the SPARC study. Stroke Prevention: Assessment of Risk in a Community. Mayo Clin Proc 74:862-869

35. Lacomis JM, Goitein O, Deible C et al (2007) Dynamic multidimensional imaging of the human left atrial appendage. Europace 9:1134-1140

36. Beigel R, Wunderlich NC, Ho SY, Arsanjani R, Siegel RJ (2014) The left atrial appendage: anatomy, function, and noninvasive evaluation. JACC Cardiovasc Imaging 7:1251-1265

37. Di Biase L, Santangeli P, Anselmino M et al (2012) Does the left atrial appendage morphology correlate with the risk of stroke in patients with atrial fibrillation? Results from a multicenter study. J Am Coll Cardiol 60:531-538

38. Cameli M, Caputo M, Mondillo S et al (2009) Feasibility and reference values of left atrial longitudinal strain imaging by twodimensional speckle tracking. Cardiovasc Ultrasound 7:6

39. Guglielmo M, Baggiano A, Muscogiuri G et al (2019) Multimodality imaging of left atrium in patients with atrial fibrillation. $\mathrm{J}$ Cardiovasc Comput Tomogr 13:340-346

40. Corradi D, Callegari S, Maestri R, Benussi S, Alfieri O (2008) Structural remodeling in atrial fibrillation. Nat Clin Pract Cardiovasc Med 5:782-96

41. Ausma J, Wijffels M, Thoné F, Wouters L, Allessie M, Borgers M (1997) Structural changes of atrial myocardium due to sustained atrial fibrillation in the goat. Circulation 96:3157-63
42. Pathak R, Lau DH, Mahajan R, Sanders P (2013) Structural and Functional Remodeling of the Left Atrium: Clinical and Therapeutic Implications for Atrial Fibrillation. J Atr Fibrillation 6(4):986

43. Yoon YE, Oh IY, Kim SA et al (2015) Echocardiographic Predictors of Progression to Persistent or Permanent Atrial Fibrillation in Patients with Paroxysmal Atrial Fibrillation (E6P Study). J Am Soc Echocardiogr 28:709-717

44. Nattel S, Harada M (2014) Atrial remodeling and atrial fibrillation: recent advances and translational perspectives. J Am Coll Cardiol 63:2335-45

45. Benussi S, de Maat GE (2018) Atrial remodelling and function: implications for atrial fibrillation surgery. Eur J Cardiothorac Surg 53:i2-i8

46. Pedersen OD, Køber L, Torp-Pedersen C (2004) Atrial fibrillation and atrial cardiomyopathy-two sides of the same coin? Am Heart J 147:953-955

47. Kadappu KK, Abhayaratna K, Boyd A et al (2016) Independent echocardiographic markers of cardiovascular involvement in chronic kidney disease: the value of left atrial function and volume. J Am Soc Echocardiogr 29:359-367

48. Kuppahally SS, Akoum N, Burgon NS et al (2010) Left atrial strain and strain rate in patients with paroxysmal and persistent atrial fibrillation: relationship to left atrial structural remodeling detected by delayed-enhancement MRI. Circ Cardiovasc Imaging 3:231-239

49. Marrouche NF, Wilber D, Hindricks G et al (2014) Association of atrial tissue fibrosis identified by delayed enhancement MRI and atrial fibrillation catheter ablation: the DECAAF study. JAMA 311:498-506

50. Saha SK, Kiotsekoglou A (2018) Value of speckle tracking echocardiography for prediction of stroke risk in atrial fibrillation: Time to spare a stare outside the box? Echocardiography 35:589-91

51. Klein AL, Murray RD, Black IW et al (1996) Integrated backscatter for quantification of left atrial spontaneous echo contrast. J Am Coll Cardiol 28:222-31

52. Ito T, Suwa M, Nakamura T, Miyazaki S, Kobashi A, Kitaura Y (2001) Quantification of left atrial appendage spontaneous echo contrast in patients with chronic nonvalvular atrial fibrillation. J Cardiol 37:325-33

53. Melillo E, Palmiero G, Ferro A, Mocavero PE, Monda V, Ascione L (2019) Diagnosis and Management of Left Atrium Appendage Thrombosis in Atrial Fibrillation Patients Undergoing Cardioversion. Medicina (Kaunas) 55:511

Publisher's Note Springer Nature remains neutral with regard to jurisdictional claims in published maps and institutional affiliations. 